\section{Hereditäres Angioödem - Frühe Behandlung verkürzt Attacken-Dauer}

D as noch immer relativ unbekannte hereditäre Angioödem (HAE) ist durch Schwellungen an Haut und Schleimhäuten charakterisiert, die im Kopf-Hals-Bereich lebensbedrohlich sein können. Die neue internationale Leitlinie der World Allergy Organization (WAO) empfiehlt unter anderem den möglichst frühzeitigen Einsatz des vom Patienten selbst applizierbaren Bradykinin-B2Rezeptorantagonisten Icatibant [WAO Journal 2012; 5: 182-99]. „Auch wenn das HAE eine seltene Erkrankung ist, sollten Hausärzte, Allergologen, Dermatologen, HNO-Ärzte und Notfallmediziner es kennen“, betonte Prof. Dr. Marcus Maurer, Charité - Universitätsmedizin Berlin. Treten die Attacken im Magen-Darm Trakt auf, kommt es häufig zu Fehldiagnosen und nicht-indizierten Operationen. Bei Schwellungen im Kopf-Hals-Bereich besteht Lebensgefahr: „Jeder HAEPatient ist lebenslänglich in Gefahr, eine Attacke der oberen Atemwege zu erleiden und daran zu ersticken“, so Maurer.
Dr. Emel Aygören-Pürsün, Universitätsklinikum Frankfurt a. M., verwies auf die hohe Verwechslungsgefahr des HAE mit allergisch bedingten Schwellungen. Beim HAE ist jedoch eine erhöhte Bradykinin-Konzentration die Ursache für die gesteigerte Gefäßpermeabilität. Als Ursache wurde bei den Formen HAE-1 und -2 ein quantitativer oder funktioneller C1-Inhibitor-Mangel identifiziert. Bekannt ist außerdem das HAE-3 ohne C1-Inhibitor-Mangel, das mit oder ohne Mutationen im Gen des Gerinnungsfaktors XII vorliegen kann. Erworbene Formen der Bradykinin-vermittelten Erkrankung sind der erworbene C1-Inhibitor-Mangel und das ACEHemmer-induzierte Angioödem.

Typisch für HAE ist, dass Symptome wie Juckreiz, Erytheme und Urtikaria meist nicht vorkommen und es auf Antihistaminika und Glukokortikoide nicht anspricht. Für eine effektive Therapie des HAE ist eine labordiagnostische Abklärung durch Bestimmung der C1-In-
hibitor-Konzentration bzw. -aktivität, der C4-Komplement- und der C1q-Spiegel notwendig.

Als effektive Behandlungsoptionen stehen die subkutane Therapie mit dem Bradykinin-B2-Rezeptor-Antagonisten Icatibant $\left(\right.$ Firazyr $\left.^{\circledast}\right)$ oder die intravenöse Substitution mit C1-Inhibitor-Konzentraten zur Verfügung. Da Icatibant als Fertigspritze zur Selbstanwendung zugelassen ist, kann sich der Patient einfach und schnell auch unter psychologischem Druck, wie z. B. bei einer Larynxattacke, selbst behandeln. Einer aktuellen Studie zufolge verkürzt eine rasche Behandlung nachweislich die Attackendauer [Maurer et al. PLoS ONE 2013: 8,e53773].

Weitere Informationen zum HAE liefert die Neuauflage der Broschüre „Heriditäres Angioödem: Update Differentialdiagnose, Therapie und Studiendaten“, die sich in erster Linie an Mediziner richtet und per E-Mail an uschirk@shire.com bestellt werden kann.

Dr. Claudia Bruhn

Presseroundtable "5 Jahre Firazyr ${ }^{\circledast}$ (Icatibant) haben die Therapie des hereditären Angioödems verändert", Berlin, 7. Oktober 2013; Veranstalter: Shire

\title{
Effektive Kurzzeitimmuntherapie bei saisonaler allergischer Rhinitis
}

\begin{abstract}
— ine präsaisonale Kurzzeitimmunthe— rapie mit nur vier Injektionen eines mit MPL (Monophosphoryl-Lipid-A) adjuvantierten Allergoids (Ragweed MATA MPL; Pollinex ${ }^{\circledast}$ Quattro Ragweed) lindert die Symptome bei saisonaler allergischer Rhinokonjunktivitis (SAR) aufgrund ei-
\end{abstract}

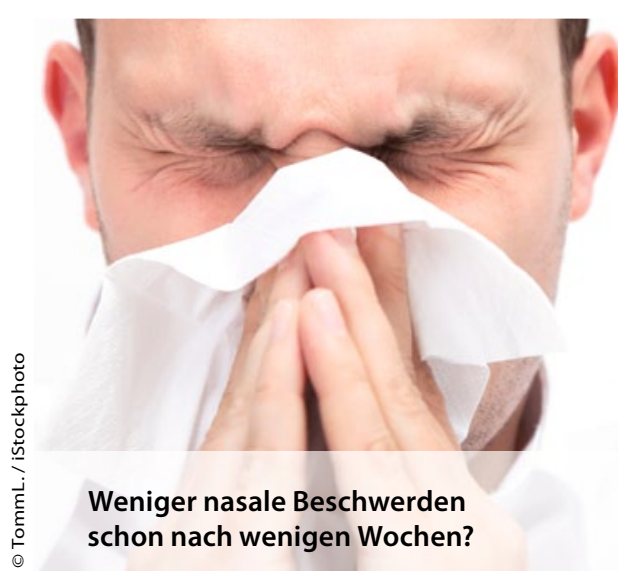

ner Ragweedpollen-Sensibilisierung innerhalb kurzer Zeit signifikant. So lautet das Ergebnis einer Studie, die randomisiert, placebokontrolliert und doppelblind die klinische Wirksamkeit und die Verträglichkeit von Ragweed MATA MPL untersucht hat, und zwar bei Patienten (18 bis 65 Jahre) mit mäßig bis starker SAR, hervorgerufen durch Ragweed-Pollen [Patel P et al. J Allergy Clin Immunol 2013 Jul 16]. Jeder Teilnehmer erhielt vier Injektionen Ragweed MATA MPL ( $n=95)$ subkutan in steigender Dosisstärke oder Placebo ( $n=93)$, jeweils im Abstand von einer Woche. Die klinische Wirksamkeit wurde anhand des TSS (Total Symptom Score) für nasale und nicht nasale Beschwerden bei Exposition in einer Pollenexpositionskammer ermittelt.

Die Kurzzeitimmuntherapie reduzierte die pollenassoziierten allergischen Beschwerden im Vergleich zum Ausgangs- wert signifikant besser als Placebo - mit einem Vorteil von $42 \%$. Verglichen mit Placebo lag die relative Überlegenheit bei $48 \%$. Auch die Lebensqualität der Patienten, ermittelt anhand des RQLQ-EEC (Rhinoconjunctivitis Quality of Life Questionnaire for use in the EEC), stieg an. Der Blick auf die Nebenwirkungen zeigt: Die Patienten klagten nach VerumInjektion etwas häufiger über lokale Reaktionen an der Injektionsstelle (90\% vs. $70 \%)$. Systemische Begleiterscheinungen waren v. a. Kopfschmerzen und seltener Schnupfen, beides lag aber auf Placeboniveau. Schwere Nebenwirkungen traten nicht auf. Damit erlaubt Ragweed MATA MPL eine Kurzzeitimmuntherapie, die die typischen Symptome der SAR bereits nach vier Injektionen innerhalb von drei Wochen signifikant reduziert. Entscheidend dafür ist der Zusatz des Adjuvans MPL.

Dr. Beate Fessler

Symposium „SIT - 100 Jahre und nichts Neues?" im Rahmen des 8. Deutschen Allergiekongresses, Bochum, 6. September 2013; Veranstalter: Bencard 